\title{
ASYMMETRIC DETRENDED FLUCTUATION ANALYSIS REVEALS ASYMMETRY IN THE RR INTERVALS TIME SERIES
}

\author{
Dawid Mieszkowski ${ }^{1}$, Marcin Kośmider ${ }^{1}$, Tomasz Krauze ${ }^{2}$ \\ Przemysław Guzik ${ }^{2}$, Jarosław Piskorski ${ }^{1}$ \\ ${ }^{1}$ Institute of Physics, University of Zielona Gora \\ Zielona Góra, Poland \\ ${ }^{2}$ Department of Cardiology - Intensive Therapy, University of Medical Sciences \\ Poznań, Poland \\ dawid.mieszkowski@gmail.com,kosmo@codelab.pl,tomaszkrauze@wp.pl \\ pguzik@ptkardio.pl,jaropis@zg.home.pl
}

\begin{abstract}
In this paper we apply the Asymmetric Detrended Fluctuation Analysis to the $R R$ intervals time series. The mathematical background of the ADFA method is discussed in the context of heart rate variability and heart rate asymmetry. We calculate the $\alpha^{+}$and $\alpha^{-}$ ADFA scaling exponents for $100 R R$ intervals time series recorded in a group of healthy volunteers (20-40 years of age) with the use of the local ADFA. It is found that on average $\alpha^{+}<\alpha^{-}$, and that locally $\alpha^{-}$dominates most of the time over $\alpha^{+}$- both results are highly statistically significant.
\end{abstract}

Keywords: scaling exponents, random walk, detrended fluctuation analysis, heart rate variability, heart rate asymmetry

\section{Introduction}

The $R R$ intervals time series, which is the time series of time intervals between successive R-waves (see Fig. 1), is perhaps one of the most often studied signals of physiological origin. It is easily obtainable and its structure lends itself well to various existing time-series analysis methods.

The most often used approach to $R R$ intervals time series is the heart rate variability (HRV) analysis which explores the changes of this series in time and tries to relate the results to various physiological or pathological states [1].

The $R R$ intervals time series is nonstationary in the sense that it does not have well-defined moments [1]. Consequently, many of the HRV methods can only be related to the specific analysed series since quantities like mean or variance cannot be related to the underlying generating process.

In recent years there have been many attempts to deal with the nonstationarity problem - one of them is the detrended fluctuation analysis (DFA) [2, 3], another one is the Heart Rate Asymmetry (HRA) analysis [4,5]. The former analyses 
the correlational structure of the noise remaining after detrending the time series, the latter concentrates on the quantification and qualification of the systematic differences between heart rate accelerations and decelerations in the variance, structure and complexity spaces.

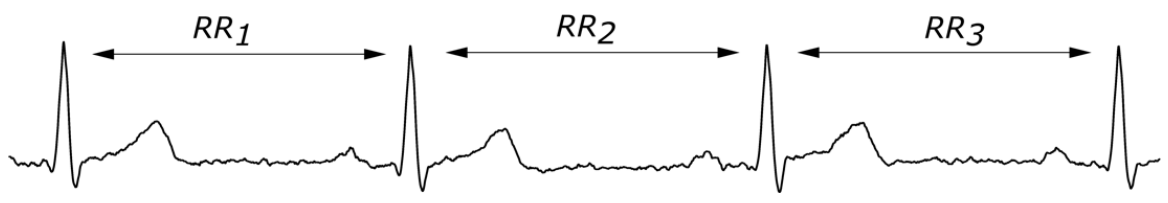

Fig. 1. Formation of the $R R$ intervals time series as the sequence of time distances between consecutive heart beats (distances between the consecutive R-waves)

A recent method of Asymmetric Detrended Fluctuation Analysis (ADFA) seems to be capable of summarizing both the correlational structure of the $R R$-intervals time series and the differences between accelerating and decelerating segments $[6,7]$.

The aim of this paper is to apply the ADFA technique to the $R R$ intervals time series in order to see whether this asymmetric technique also reveals the asymmetry inherent in the heart rate.

\section{Detrended fluctuation analysis}

The method of detrended fluctuation analysis studies the random walk defined on the analysed time series. The basic idea is to calculate the variance of the random walk at a number of scales and study the relationship between the variance and scale. For example, for a random walk defined by a coin flip we have

$$
\sigma \sim \mathrm{N}^{\frac{1}{2}}
$$

where $\mathrm{N}$ is the number of steps of length 1 in the random walk [8]. This is an example of a more general type of statistical property of a time series, namely

$$
\sigma \sim \mathrm{N}^{\alpha}
$$

where $\alpha$ is the universal critical exponent which is characteristic of a power law observed in scale-invariant systems [8].

The above analysis can be applied to a number of correlated or uncorrelated types of noise. The $R R$-intervals time series, however, is non-stationary as it contains trends. The detrended fluctuation analysis aims at defining a "random walk" on this time series by removing these trends and analysing the resulting noise.

This is achieved by defining the root mean square fluctuation of the random walk 


$$
\mathrm{F}(\mathrm{n})=\sqrt{\frac{1}{\mathrm{n}} \sum_{\mathrm{k}=1}^{\mathrm{N}} \epsilon_{\mathrm{k}}^{2}}
$$

where $\epsilon_{\mathrm{k}}^{2}$ is defined as an integrated and detrended time series as follows.

First, let us define the original time series as

$$
\mathbf{R R}=\left\{\mathrm{RR}_{\mathrm{i}}\right\}_{\mathrm{i}=1}^{\mathrm{N}} \text {, }
$$

i.e. it is a time series of $\mathrm{N}$ consecutive $R R$ intervals indexed by the number of the cardiac evolutions.

The mean of this time series is different from zero, so the random walk defined on it would contain drift, therefore, before integrating, the mean is subtracted

$$
y(k)=\sum_{i=1}^{k}\left(R R_{i}-\overline{R R}\right),
$$

where $\overline{\mathrm{RR}}$ is the mean of the time series $[2,3]$.

This integrated time series is divided into segments of constant length $\mathrm{n}$ and in each segment a straight line is fitted

$$
\mathrm{y}_{\mathrm{n}}(\mathrm{k})=\mathrm{ak}+\mathrm{b}, \quad \mathrm{k}=1 \ldots \mathrm{n},
$$

and finally the fluctuation $\epsilon_{\mathrm{k}}$ used in equation (3) is defined as

$$
\epsilon_{\mathrm{k}}=\mathrm{y}(\mathrm{k})-\mathrm{y}_{\mathrm{n}}(\mathrm{k}),
$$

i.e. the local trends are removed from the integrated time series $[2,3,6,7]$. The $a$ and $b$ parameters may be fitted with any optimization technique like, for example, the least squares method.

$\mathrm{F}(\mathrm{n})$ defined in equation (3) is now calculated for all time scales available in the time series and the power law relationship between this fluctuation and box size is explored $[2,3]$. If

$$
\mathrm{F}(\mathrm{n}) \sim \mathrm{n}^{\alpha},
$$

holds, we may conclude the existence of the power law and, consequently, the existence of long-range correlations in the time series $[2,3,8]$.

It is worth noting that the number of scales necessary for $F(n)$ calculation can be greatly reduced by using the approach suggested by Peng et al. in [9].

The value of the $\alpha$ exponent, often called scaling exponent, is derived by fitting a straight line to the $\log _{10} \mathrm{~F}(\mathrm{n})$ on $\log _{10}(\mathrm{n})$ plot. This exponent is used to classify the analysed noise $[2,3]$ : 
- $0<\alpha<0.5$ - negative long-range correlations,

- $\alpha=0.5$ - white noise with no correlations,

- $0.5<\alpha<0.1$ - positive long-range correlations,

- $\alpha=1-1 / \mathrm{f}$ noise, typical of many biological time series,

- $1<\alpha<1.5$ - positive long-range correlations which do not follow the power law,

- $\alpha=1.5-$ red (Brownian) noise.

Figure 2 presents the DFA of one of the recordings used in this paper. It can be seen that the $\alpha$ exponent is consistent with 1 , so it can be concluded that there is $1 / \mathrm{f}$ noise in the analysed signal.

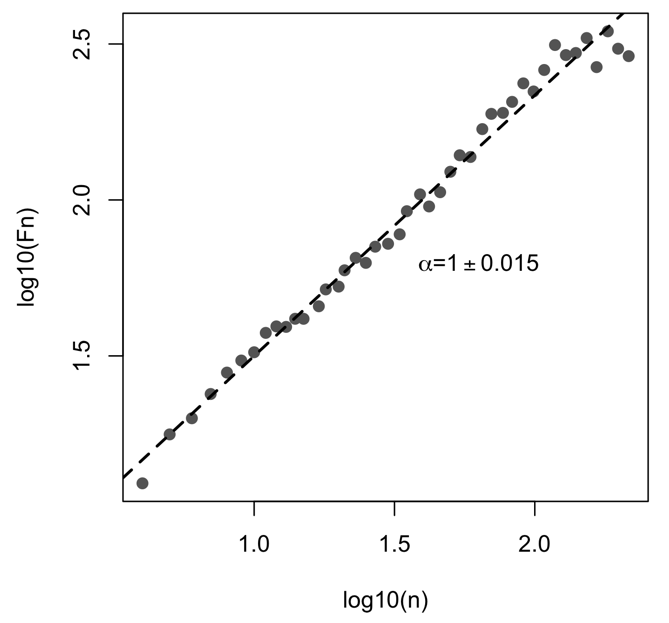

Fig. 2. Example DFA analysis of an $R R$-intervals time series from a 33 year-old male

\section{Asymmetric detrended fluctuation analysis}

In recent years there has been a lot of research on the asymmetric properties of the $R R$ intervals time series $[4,5,10]$. The reason for this is the fact that the accelerations and decelerations of heart rate are well-defined physiological processes, even though the specific mechanisms that govern them are very complex. It has been demonstrated that parameters describing the asymmetric behaviour of the $R R$ intervals time series have prognostic value in the sense that the prognosis of the patient, like for instance the probability of surviving the next two years after myocardial infarction, can be informed by analysing the asymmetric parameters [10].

At the same time, for different reasons, the DFA has been extended to the asymmetric case and the asymmetric detrended fluctuation analysis (ADFA) approach was created $[6,7]$. The motivation for doing so may be found in $[6,7]$. This modification consists of two steps. First, the time series are divided into 
segments with rising and falling trends (on the basis of the sign of the a statistic compare eq. (6)). Then fluctuations on raising and falling trends are defined separately as

$$
\mathrm{F}^{+}(\mathrm{n})=\sqrt{\frac{1}{\mathrm{M}_{\mathrm{n}}^{+} \cdot \mathrm{n}} \sum_{\mathrm{k}=1}^{\mathrm{M}_{\mathrm{n}}^{+}}\left(\mathrm{y}(\mathrm{k})-\mathrm{y}_{\mathrm{n}}(\mathrm{k})\right)^{2}}
$$

for rising trends, and

$$
\mathrm{F}^{-}(\mathrm{n})=\sqrt{\frac{1}{\mathrm{M}_{\mathrm{n}}^{-} \cdot \mathrm{n}} \sum_{\mathrm{k}=1}^{\mathrm{M}_{\mathrm{n}}^{-}}\left(\mathrm{y}(\mathrm{k})-\mathrm{y}_{\mathrm{n}}(\mathrm{k})\right)^{2}},
$$

for falling trends. $\mathrm{M}_{\mathrm{n}}^{ \pm}$is the number of boxes of length $\mathrm{n}$ with rising/falling trend, respectively, [7].

Asymmetric scaling exponents are defined as

$$
\mathrm{F}^{ \pm}(\mathrm{n}) \sim \mathrm{n}^{\alpha^{ \pm}}
$$

provided that power law holds $[6,7]$. Similarly to the standard DFA, the $\alpha^{ \pm}$exponents are derived from the plots of $\log _{10} \mathrm{~F}^{ \pm}(\mathrm{n})$ on $\log _{10}(\mathrm{n})[6,7]$.

Another modification concerns the mode of calculating the asymmetric exponents. It is possible to calculate them for the entire recording with $R R$ intervals, however, since the very long trends present in the $R R$ intervals time series can be ascribed to activity or circadian rhythms, which are not part of the autonomic system modulation (they can influence it, though), it seems reasonable to concentrate on shorter scale trends. Another reason is the fact that the acceleration and deceleration runs, which form the basis of one of the more successful approaches to HRA, are observable on a short scale, the longest being the acceleration runs of length approximately 20 beats $[5,10]$.

A localized version of ADFA was developed in [7]. It boils down to calculating the $\alpha^{ \pm}$exponents in sliding or jumping windows, in the original paper the length of this window was selected to be 100 samples long, and producing two derivative time series of $\alpha^{+}$and $\alpha^{-}$.

\section{Materials and methods}

The studied group consisted of 100 healthy volunteers, 45 women, age range 20-40 years. In each subject standard 30-minute, 12-lead ECG was recorded at $1600 \mathrm{~Hz}$. The ECG curves were registered by the analog-digital converter (Porti 5, TMSI, The Netherlands) and saved to the hard disk. The post-processing was 
carried out with the libRASCH/RASCHlab (v. 0.6.1, Raphael Schneider, Germany). The automatic classification into beats of sinus, ventricular, supraventricular and artifact was verified by an expert who edited the classification as the need arose. The $R R$ intervals with annotations were exported to text files which were further analysed by the in-house software written in python and cython for dynamic ADFA (the git repository for this software with complete code can be found at https://github.com/kosmo76/adfa). The analysis used a 100 long sliding window.

The results were analysed with the use of the $\mathrm{R}$ statistical package and programming language. For each recording the average and standard deviation were calculated, and these results were used to calculate the mean and standard deviation weighted by the variances of each recording, according to

$$
\overline{\alpha^{ \pm}}=\frac{\sum_{\mathrm{i}=1}^{100}\left(\alpha_{\mathrm{i}}^{ \pm} \sigma_{\mathrm{i}}^{-2}\right)}{\sum_{\mathrm{i}=1}^{100} \sigma_{\mathrm{i}}^{-2}} ; \quad \sigma_{\overline{\alpha^{ \pm}}}^{2}=\frac{1}{\sum_{\mathrm{i}=1}^{100} \sigma_{\mathrm{i}}^{-2}}
$$

and these values were reported.

After checking for normality of the distribution of the $\alpha^{ \pm}$, they were used for the paired $t$-test for equality of the means. The resulting $\alpha^{ \pm}$time series were also analysed for the time during which $\alpha^{+}<\alpha^{-}$and vice versa. Since for random data the times should be equal, the binomial test was used to establish whether any of the above inequalities clearly dominates.

\section{Results}

The mean value of $\alpha^{+}$was $0.89 \pm 0.02$, the mean value of $\alpha^{-}$was $0.98 \pm 0.02$. The comparison with the paired $t$-test yields $p<0.0001$, which means that there is a highly statistically significant difference between $\alpha^{+}$, and $\alpha^{-}$, with $\alpha^{+}<\alpha^{-}$. Both the distribution of the $\alpha^{ \pm}$values and the paired $t$-test are illustrated in Figure 3 .
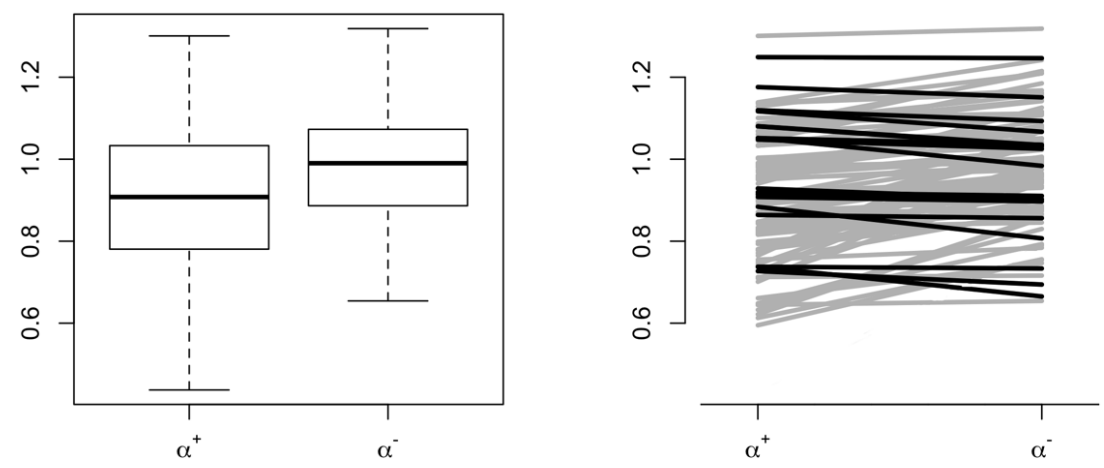

Fig. 3. Comparison of the $\alpha^{+}$and $\alpha^{-}$scaling exponents. The left panel presents the boxplots of these exponents, the right panel demonstrates the paired $t$-test comparison. The lines connect $\alpha^{+}$and $\alpha^{-}$scaling exponents from the same recording - grey corresponds to $\alpha^{+}<\alpha^{-}$and black to the reversed inequality 
Another way in which the asymmetry can be observed in ADFA is counting the time during which $\alpha^{+}<\alpha^{-}$. Overall, of the 100 analysed recordings, in 82 we observed $\alpha^{+}<\alpha^{-}$, in the remaining ones the inequality was reversed. It is interesting to note that this result is similar to the percentages obtained for variance-based asymmetry descriptors [4]. This result can be analysed with the use of the binomial test: if the time series was symmetric, there should be no differences between upward and downward trends, so the proportion should be 0.5 rather than the observed 0.8. The binomial test shows that the difference between the obtained result and the symmetric result is highly statistically significant with $p<0.0001$. This analysis is illustrated in Figure 3 above.

\section{Conclusions}

In the present paper we have applied the ADFA technique to short, 30-minute long, stationary recordings. We have used the local version of ADFA which utilizes jumping, 100-beat window and calculates the scaling exponents $\alpha^{+}$and $\alpha^{-}$for each window, thus creating a derivative, multivariate time series.

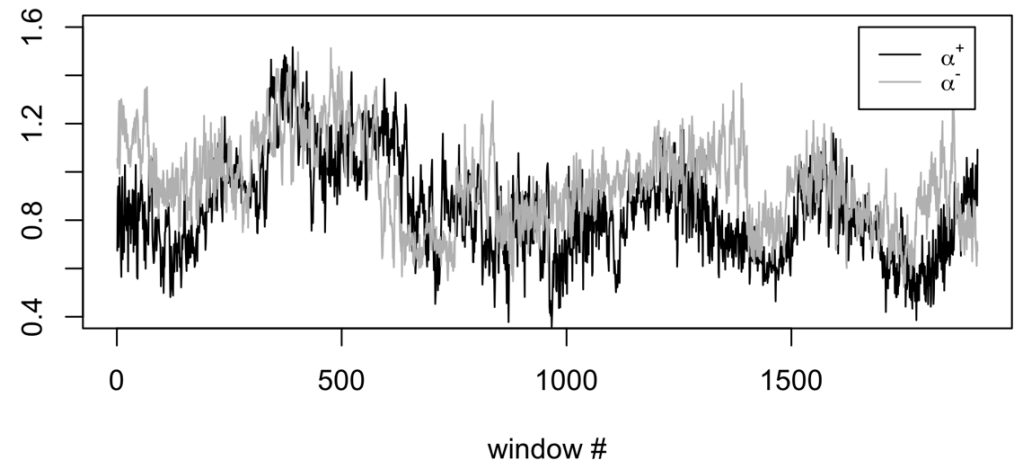

Fig. 4. Example of the local value of the $\alpha^{+}$and $\alpha^{-}$scaling exponents in one recording. It is evident that most of the time there is $\alpha^{+}<\alpha^{-}$

The result obtained was that there is a clear and easily observable asymmetry in the correlational structure of the $R R$ intervals time series. We observed that, averaged over all the local windows, there is $\alpha^{+}<\alpha^{-}$, and this observation is highly statistically significant. Also, inequality of the same direction is observable in the time in which it is fulfilled: in $80 \%$ of the recordings, for the majority of time, there was $\alpha^{+}<\alpha^{-}$. This result is also highly statistically significant.

HRA was first observed using the variance based descriptors and then expanded upon with the use of the method of accelerations and decelerations runs $[4,5,10]$. The main mechanism in which HRA arises mathematically is the fact that decelerations seem to be more dynamic than accelerations in the sense that fewer steps are needed to decelerate by, say, $50 \mathrm{bpm}$ (beats per minute), than to accelerate by the same amount $[5,10]$. The original papers introducing ADFA aimed at describ- 
ing economic time series $[6,7]$, in which collapses are much more dynamic than recoveries. It is very possible that the fact that asymmetry is also visible in the $R R$ intervals follows from this similarity between the two types of time series.

One limitation of the method used in the present paper could be raised here. The ADFA practically treats falling and rising trends as if they were coming from two separate time series or two separate processes. In the case of heart rate it is very difficult to say with certainty what caused an acceleration or deceleration. The dynamic balance between the parasympathetic and sympathetic branches of the autonomic nervous system is responsible for the instant changes of heart rate that can be observed in a form of accelerations and decelerations. The widespread belief that it is the parasympathetic branch of the autonomic system which is responsible for decelerations and the sympathetic branch which is responsible for accelerations is only a first approximation and in reality these processes are much more complex.

Nevertheless, ADFA is a new and promising method of exploring the inherent asymmetry of the $R R$ intervals time series. Further studies, both theoretical and data-based are necessary to understand both the mathematical structure of the method and its results when applied to the very important and very complex RR intervals time series.

\section{References}

[1] Sassi R., Cerutti S., Lombardi F., Malik M., Huikuri H.V., Peng C.K., Schmidt G., Yamamoto Y., Advances in heart rate variability signal analysis: Joint position statement by the e-Cardiology ESC Working Group and the European Heart Rhythm Association co-endorsed by the Asia Pacific Heart Rhythm Society, Europace 2015, 17, 1341-1353.

[2] Peng C.K., Buldyrev S.V., Havlin S., Simons M., Stanley H.E., Goldberger A.L., Mosaic organization of DNA nucleotides, Phys. Rev. E 1994, 49, 1685-1689.

[3] Peng C.K., Havlin S., Stanley H.E., Goldberger A.L., Quantification of scaling exponents and crossover phenomena in nonstationary heartbeat time series, Chaos 1995, 5, 82-87.

[4] Piskorski J., Guzik P., Geometry of the Poincaré plot of RR intervals and its asymmetry in healthy adults, Physiol. Meas. 2007, 28, 287-300.

[5] Piskorski J., Guzik P., The structure of heart rate asymmetry: deceleration and acceleration runs, Physiol. Meas. 2011, 32, 1011-1023.

[6] Ramirez J.A., Rodrigues E., Echeverria J.C., A DFA approach for assessing asymmetric correlations, Physica A 2009, 388, 2263-2270.

[7] Rivera-Castro M.A., Miranda J.G.V., Cajueiro D.O., Andrade R.F.S., Detecting switching points using asymmetric detrended fluctuation analysis, Physica A 2012, 391, 170-179.

[8] Sethna J.P., Statistical Mechanics: Entropy, Order Parameters and Complexity, Oxford University Press, Oxford 2006.

[9] Goldberger A.L., Amaral L.A.N., Glass L., Hausdor J.M., Ivanov P.Ch., Mark R.G., Mietus J.E., Moody G.B., Peng C.K., Stanley H.E., PhysioBank, Physio-Toolkit, and Physionet: Components of a new research resource for complex physiologic signals, Circulation 2001, 101, e215-e220.

[10] Guzik P., Piskorski J., Barthel P., Bauer A., Müller A., Junk N., Ulm K., Malik M., Schmidt G., Heart rate deceleration runs for postinfarction risk prediction, J. Electrocardiol. 2012, 45, 70-75. 